\title{
A Mixed $H_{2} / H_{\infty}$-Based Semiactive Control for Vibration Mitigation in Flexible Structures
}

\author{
Mauricio Zapateiro, Hamid Reza Karimi, Ningsu Luo, Brian M. Phillips and Billie F. Spencer, Jr.
}

\begin{abstract}
In this paper, we address this problem through the design of a semiactive controller based on the mixed $H_{2} / H_{\infty}$ control theory. The vibrations caused by the seismic motions are mitigated by a semiactive damper installed in the bottom of the structure. It is meant by semiactive damper, a device that absorbs but cannot inject energy into the system. Sufficient conditions for the design of a desired control are given in terms of linear matrix inequalities (LMIs). A controller that guarantees asymptotic stability and a mixed $H_{2} / H_{\infty}$ performance is then developed. An algorithm is proposed to handle the semiactive nature of the actuator. The performance of the controller is experimentally evaluated in a real-time hybrid testing facility that consists of a physical specimen (a small-scale magnetorheological damper) and a numerical model (a large-scale three-story building).
\end{abstract}

\section{INTRODUCTION}

In recent years, the protection of structures against hazardous vibrations has gained special interest. Structures such as buildings, bridges and vehicle suspension systems are subject to vibrations that may cause malfunctioning, uncomfort or collapse. It is an extended practice to install damping devices in order to mitigate such vibrations [1]. Because of their ability in online tuning, its inherent stability and low energy requirements, semiactive devices such as magnetorheological dampers are an attractive solution and efforts have been devoted to their understanding in the past years [2], [3].

Most of semiactive structural control strategies are based on the idea of attenuating vibrations or maintaining structural time response within certain acceptable ranges, when external forces such as earthquakes or strong winds act on the structures. The controller design is usually done in time-domain by considering that the system model and its associated parameters are known or uncertain but with known upper and lower bounds. Examples of techniques used are Lyapunov theory [4], Bang-Bang control [5], Sliding Mode Control [6], and Backstepping [7]. There are, however, other works that consider the frequency modes. The modal frequency control is of a great interest for achieving the structural safety and human comfort. Examples of frequency control techniques

M. Zapateiro and N. Luo are with the Institute of Informatics and Applications, University of Girona, 17071 Girona, Spain \{mauricio.zapateiro, ningsu.luo, \}audg.edu

H.R. Karimi is with the Faculty of Technology and Science, University of Agder, Grimstad, Norway. hamid.r.karimi @uia.no

B.M. Phillips and B.F. Spencer, Jr. are with the Department of Civil and Environmental Engineering, University of Illinois at Urbana-Champaign, Urbana, IL 61801, USA. \{bphil2, bfs\}@uiuc.edu employed for mitigating vibrations are QFT [3] and $H_{\infty}[8]$

In this paper, we consider the vibration problem from a mixed time-frequency domain perspective. It is desirable that this problem be solved not only by reducing the time response but the frequency response as well. This can be approached by the mixed $H_{2} / H_{\infty}$ control methodology. The objective is to find an $H_{\infty}$ controller that achieves the robust performance of the system by minimizing its controlled output response against the external disturbances within the frequency range, while combing it with the $\mathrm{H}_{2}$ control approach in order to reduce the structural time response and control effort. Also, the controller is formulated by feeding back the output so that the limited measurements problem can be accounted for. Based on the Lyapunov theory, some required sufficient conditions are established in terms of linear matrix inequalities (LMIs) for the stability and stabilization of the considered system using some free matrices. The desired robust mixed $H_{2} / H_{\infty}$ output feedback control is derived based on a convex optimization method such that the resulting closed-loop system is asymptotically stable and satisfies $H_{2}$ performance with a guaranteed cost and a prescribed level of $H_{\infty}$ performance, simultaneously. An algorithm is also proposed to include the dynamics of the actuator in order to estimate the control signal.

This paper is organized as follows. Section II presents the problem of vibration mitigation in a $n$-story building. A detail description of the structure is presented and diverse issues for control formulation are discussed. Section III is devoted to the details of the formulation of the output feedback mixed $H_{2} / H_{\infty}$ controller. LMIs for the $H_{2}$ and $H_{\infty}$ performances are developed. These LMIs form the set that solves the problem of the mixed constraints. Then, in Section IV, experimental validation of the controllers on a hybrid-testing facility is discussed. Finally, the conclusions are outlined at the end of the paper.

\section{PRoblem DEFinition}

Consider an $n$-story building with an actuator placed at the first floor, as shown in Fig. 1. This is a flexible structure that can be modeled with the second order motion equation:

$$
\mathbf{M} \ddot{\mathbf{x}}_{\mathbf{r}}+\mathbf{C} \dot{\mathbf{x}}_{\mathbf{r}}+\mathbf{K} \mathbf{x}_{\mathbf{r}}=G_{s} f_{m r}-\mathbf{M} L_{s} \ddot{x}_{g}
$$

where $x_{r}$ is the vector of relative displacements, i.e., measured with respect to the ground (the $r$ subindex means relative coordinates); $f_{m r}$ is the damper force input; $\ddot{x}_{g}$ is the input disturbance, i.e., the incoming earthquake acceleration. 


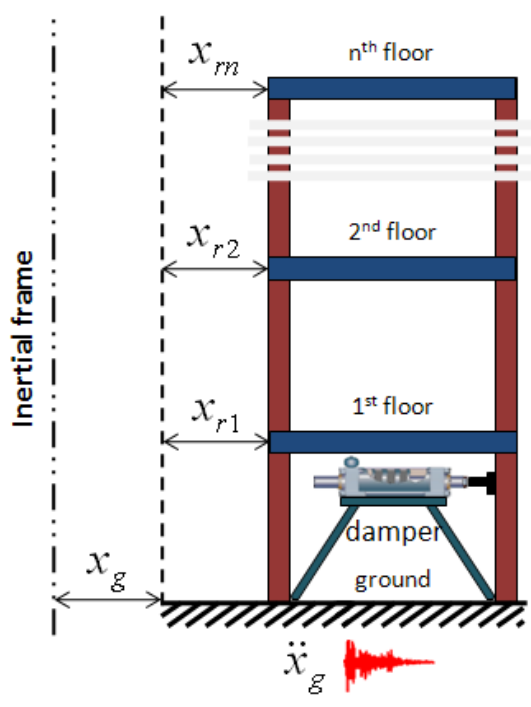

Fig. 1. Schematic of the 3-story building with MR damper.

Denote as $\ddot{x}_{a i}$ the absolute acceleration of the $i$-th floor, i.e, measured with respect to an inertial frame (the $a$ subindex means absolute coordinates). Then the relationship between relative and absolute displacements is $x_{r i}=x_{a i}-x_{g} . \mathbf{M}_{\mathbf{s}}$ is the mass matrix, $\mathbf{C}_{\mathbf{s}}$ is the damping matrix, $\mathbf{K}_{\mathbf{s}}$ is the stiffness matrix, $G_{s}$ is a vector that accounts for the location of the damping devices in the structure and $L_{s}$ is a vector that accounts for the disturbance inputs. The structure of these matrices and vectors is:

$$
\begin{gathered}
\mathbf{M}_{\mathbf{s}}=\operatorname{diag}\left(m_{i}\right), i=1,2, \ldots, n \\
\mathbf{C}_{\mathbf{s}}=\left[\begin{array}{cccc}
c_{1}+c_{2} & -c_{2} & 0 & 0 \\
-c_{2} & c_{2}+c_{3} & -c_{3} & 0 \\
\vdots & \vdots & \vdots & \vdots \\
0 & 0 & -c_{n} & c_{n}
\end{array}\right] \\
\mathbf{K}_{\mathbf{s}}=\left[\begin{array}{cccc}
k_{1}+k_{2} & -k_{2} & 0 & 0 \\
-k_{2} & k_{2}+k_{3} & -k_{3} & 0 \\
\vdots & \vdots & \vdots & \vdots \\
0 & 0 & -k_{n} & k_{n}
\end{array}\right] \\
G_{s}=[-1,0, \ldots, 0]^{\mathrm{T}} \quad L_{s}=[1,1, \ldots, 1]^{\mathrm{T}}
\end{gathered}
$$

The objective of the control design is to reduce the structure response when subject to a seismic motion. Earthquakes are unpredictable events whose duration and intensity are unknown but bounded. The goal is to keep the relative displacements and the absolute accelerations as small as possible with a low control effort. In general, minimizing the relative displacement of each floor leads to preserving the integrity of the structure while reducing the absolute accelerations helps improving both the security and comfort of the occupants. Furthermore, it is desirable that the amount of sensors necessary for control implementation is as minimal as possible. Sensors usually available for controller implementation and monitoring are accelerometers, which provide a direct reading of the absolute acceleration; load cells for measuring the damper force; and linear voltage displacement transducers (LVDT) which provide a measure of the relative displacements. Accelerometers are the most widely used sensors because of practical implementation issues.

In this research, the mixed $H_{2} / H_{\infty}$ control approach is proposed to solve the vibration mitigation problem of the $n$-story structure. The structural response reduction under unknown disturbances can be achieved with the $H_{\infty}$ control while the minimization of control effort requirements can be achieved with the $H_{2}$ control. Moreover, in order to account for limited measurement availability, an output-feedback approach is chosen.

The $n$-story building dynamic model can be written in state space form as follows:

$$
\begin{gathered}
\dot{\mathbf{x}}=\mathbf{A x}+\mathbf{B}_{\mathbf{1}} \mathbf{u}+\mathbf{B}_{\mathbf{2}} \mathbf{w} \\
\mathbf{z}=\mathbf{C}_{\mathbf{1}} \mathbf{x}+\mathbf{D}_{1 \mathbf{1}} \mathbf{u}+\mathbf{D}_{\mathbf{1 2}} \mathbf{w} \\
\mathbf{y}=\mathbf{C}_{\mathbf{2}} \mathbf{x}+\mathbf{D}_{\mathbf{2 1}} \mathbf{u}+\mathbf{D}_{\mathbf{2 2}} \mathbf{w}
\end{gathered}
$$

where $\mathbf{x}=\left[\mathbf{x}_{\mathbf{r}}, \dot{\mathbf{x}}_{\mathbf{r}}\right]^{\mathrm{T}}$ is the state vector, composed of the relative displacements and velocities of each floor; $\mathbf{u}$ is the input vector, i.e., the damper force input $\left(f_{m r}\right)$; w is the exogenous input vector, i.e., the seismic motion acceleration $\left(\ddot{x}_{g}\right) ; \mathbf{z}=\left[\boldsymbol{\Lambda}_{\mathbf{1}} \mathbf{x}_{\mathbf{r}}, \boldsymbol{\Lambda}_{\mathbf{2}} \ddot{\mathbf{x}}_{\mathbf{a}}\right]^{\mathrm{T}}$ is the vector of controlled outputs which is composed of weighted relative displacements and absolute accelerations $\left(\boldsymbol{\Lambda}_{1}\right.$ and $\boldsymbol{\Lambda}_{2}$ are matrices that contain the weighting factor of the relative displacements and absolute accelerations); and $\mathbf{y}=\ddot{\mathbf{x}}_{\mathbf{a}}$ is the vector of measured outputs which consists of the absolute acceleration measurements. The matrices of the system (6) - (8) are given by:

$$
\begin{aligned}
& \mathbf{A}=\left[\begin{array}{cc}
\mathbf{0} & \mathbf{I} \\
-\mathbf{M}_{\mathbf{s}}{ }^{-1} \mathbf{K}_{\mathbf{s}} & -\mathbf{M}_{\mathbf{s}}{ }^{-1} \mathbf{C}_{\mathbf{s}}
\end{array}\right] \\
& \mathbf{B}_{1}=\left[\begin{array}{c}
\mathbf{0} \\
\mathbf{M}_{\mathbf{s}}{ }^{-1} G_{s}
\end{array}\right] \quad \mathbf{B}_{2}=\left[\begin{array}{c}
\mathbf{0} \\
-L_{s}
\end{array}\right] \\
& \mathbf{C}_{1}=\left[\begin{array}{cc}
\mathbf{0} & \boldsymbol{\Lambda}_{\mathbf{1}} \mathbf{I} \\
-\boldsymbol{\Lambda}_{\mathbf{2}} \mathbf{M}_{\mathbf{s}}^{-1} \mathbf{K}_{\mathrm{s}} & -\boldsymbol{\Lambda}_{\mathbf{2}} \mathbf{M}_{\mathbf{s}}^{-1} \mathbf{C}_{\mathbf{s}}
\end{array}\right] \\
& \mathbf{D}_{11}=\left[\begin{array}{c}
\mathbf{0} \\
\mathbf{\Lambda}_{\mathbf{2}} \mathbf{M}_{\mathbf{s}}{ }^{-1} G_{s}
\end{array}\right] \quad \mathbf{D}_{12}=\mathbf{0} \\
& \mathbf{C}_{2}=\left[\begin{array}{ll}
-\mathbf{M}_{\mathbf{s}}^{-1} \mathbf{K}_{\mathbf{s}} & -\mathbf{M}_{\mathbf{s}}^{-1} \mathbf{C}_{\mathbf{s}}
\end{array}\right] \\
& \mathbf{D}_{21}=\mathbf{M}_{\mathbf{s}}{ }^{-1} G_{s} \quad \mathbf{D}_{22}=\mathbf{0}
\end{aligned}
$$

The $\mathrm{H}_{2}$ performance measure of the system (6) - (8) is defined as:

$$
J_{2}=\int_{0}^{\infty}\left(\mathbf{x}^{\mathrm{T}} \mathbf{S}_{\mathbf{1}} \mathbf{x}+\mathbf{u}^{\mathrm{T}} \mathbf{S}_{\mathbf{2}} \mathbf{u}\right) d t
$$

where $\mathbf{w}=\mathbf{0}$ and the constant matrices $\mathbf{S}_{\mathbf{1}}$ and $\mathbf{S}_{\mathbf{2}}$ are given On the other hand, the $H_{\infty}$ performance measure is defined as:

$$
J_{\infty}=\int_{0}^{\infty}\left(\mathbf{z}^{\mathrm{T}} \mathbf{z}-\gamma^{2} \mathbf{w}^{\mathrm{T}} \mathbf{w}\right) d t
$$


where $\gamma$ is a given positive scalar. Then, the mixed $H_{2} / H_{\infty}$ performance measure is defined as:

$$
\operatorname{Min}\left\{J_{0} \mid J_{\infty}<0 \text { and } J_{2} \leq J_{0}\right\}
$$

which is equivalent to minimize the upper bound $J_{2}$ $\left(J_{0}>0\right)$ subject to $J_{\infty}<0$. The minimization of $J_{2}$ will result in the reduction of the system response and control effort while achieving $J_{\infty}<0$ will keep the system response within prescribed intervals in the presence of external disturbances.

Up to now, several control approaches has proposed to solve the mixed $H_{2} / H_{\infty}$ control problem [9] - [12]. The problem of the controller design to be addressed in this paper is formulated as follows: given the second-order linear system (6) - (8) with a prescribed level of disturbance attenuation $\gamma>0$, find a mixed $H_{2} / H_{\infty}$ output feedback control $\mathbf{u}=\mathbf{K}_{\mathbf{2} \infty} \mathbf{y}$ where $\mathbf{K}_{\mathbf{2} \infty}$ is the control gain to be determined such that:

1) the resulting closed-loop system is asymptotically stable,

2) under $\mathbf{w}=\mathbf{0}$ the $H_{2}$ performance measure satisfies $J_{2} \leq$ $J_{0}$, where the positive scalar $J_{0}$ is a guaranteed cost,

$3)$ under zero initial conditions and for all non-zero $\mathbf{w} \in L_{2}[0, \infty)$, the upper bound of $H_{2}$ satisfies $J_{\infty}<0$.

In this case, the second order linear system (6) - (8) is asymptotically stable with a mixed $H_{2} / H_{\infty}$ performance.

\section{LMI FORMULATION OF THE OUTPUT FEEDBACK CONTROLLER}

Recall the state space model (6) - (8). Then, augmenting the control $\mathbf{u}=\mathbf{K}_{\mathbf{2} \infty} \mathbf{y}$ to the system (6) - (8) results in:

$$
\begin{gathered}
\dot{\mathbf{x}}=\overline{\mathbf{A}} \mathbf{x}+\mathbf{B}_{\mathbf{2}} \mathbf{w} \\
\mathbf{z}=\overline{\mathbf{C}} \mathbf{x}+\mathbf{D}_{12} \mathbf{w} \\
\mathbf{y}=\left(\mathbf{I}-\mathbf{D}_{\mathbf{2 1}} \mathbf{K}_{\mathbf{2} \infty}\right)^{-1} \mathbf{C}_{\mathbf{2}} \mathbf{x}
\end{gathered}
$$

where $\quad \overline{\mathbf{A}}=\mathbf{A}+\mathbf{B}_{\mathbf{1}} \mathbf{K}_{\mathbf{2} \infty}\left(\mathbf{I}-\mathbf{D}_{\mathbf{2 1}} \mathbf{K}_{\mathbf{2} \infty}\right)^{-1} \mathbf{C}_{\mathbf{2}} \quad$ and $\overline{\mathbf{C}}=\mathbf{C}_{\mathbf{1}}+\mathbf{D}_{\mathbf{1 1}} \mathbf{K}_{\mathbf{2} \infty}\left(\mathbf{I}-\mathbf{D}_{\mathbf{2 1}} \mathbf{K}_{\mathbf{2} \infty}\right)^{-1} \mathbf{C}_{\mathbf{2}}$. Now, represent the system (18) - (20) in a model descriptor form as follows:

$$
\begin{aligned}
& \dot{\mathbf{x}}=\eta_{\mathbf{d}} \\
& \mathbf{0}=-\eta_{\mathbf{d}}+\overline{\mathbf{A}} \mathbf{x}+\mathbf{B}_{\mathbf{2}} \mathbf{w}
\end{aligned}
$$

Define the following Lyapunov functional:

$$
\mathbf{V}=\mathbf{x}^{\mathrm{T}} \mathbf{P}_{\mathbf{1}} \mathbf{x}:=\left[\begin{array}{ll}
\mathbf{x}^{\mathrm{T}} & \eta_{\mathbf{d}}^{\mathrm{T}}
\end{array}\right] \mathbf{T} \mathbf{P}\left[\begin{array}{c}
\mathbf{x} \\
\eta_{\mathbf{d}}
\end{array}\right]
$$

where $\mathbf{T}=\operatorname{diag}\{\mathbf{I}, \mathbf{0}\}, \mathbf{P}_{\mathbf{1}}=\mathbf{P}_{\mathbf{1}}^{\mathrm{T}}>0$ and $\mathbf{P}=$ $\left[\begin{array}{cc}\mathbf{P}_{\mathbf{1}} & \mathbf{0} \\ \mathbf{P}_{3} & \mathbf{P}_{\mathbf{2}}\end{array}\right]$, such that $P_{2}$ and $P_{3}$ are some free matrices. Differentiating $\mathbf{V}$ along the system trajectories (18) yields:

$$
\dot{\mathbf{V}}=2\left[\begin{array}{ll}
\mathbf{x}^{\mathrm{T}} & \eta_{\mathbf{d}^{\mathrm{T}}}
\end{array}\right] \mathbf{P}^{\mathrm{T}}\left\{\left[\begin{array}{cc}
\mathbf{0} & \mathbf{I} \\
\overline{\mathbf{A}} & \mathbf{I}
\end{array}\right]\left[\begin{array}{c}
\mathbf{x} \\
\eta_{\mathbf{d}}
\end{array}\right]+\left[\begin{array}{c}
\mathbf{0} \\
\mathbf{B}_{\mathbf{2}}
\end{array}\right] \underset{(24)}{\mathbf{w}}\right\}
$$

Before proceeding with the controller formulation, a lemma that will be further used is stated next.

Lemma 1 [12]. For a given $\mathbf{M} \in \mathbb{R}^{p \times n}$ with $\operatorname{rank}(\mathbf{M})=$ $p<n$, assume that $\mathbf{Z} \in \mathbb{R}^{n \times n}$ is a symmetric matrix, then there exists a matrix $\hat{\mathbf{Z}} \in \mathbb{R}^{p \times p}$ such that $\mathbf{M Z}=\hat{\mathbf{Z}} \mathbf{M}$ if and only if

$$
\begin{gathered}
\mathbf{Z}=\mathbf{V}\left[\begin{array}{cc}
\mathbf{Z}_{1} & \mathbf{0} \\
\mathbf{0} & \mathbf{Z}_{\mathbf{2}}
\end{array}\right] \mathbf{V}^{\mathbf{T}} \\
\hat{\mathbf{Z}}=\mathbf{U} \hat{\mathbf{M}} \mathbf{Z}_{\mathbf{1}} \hat{\mathbf{M}}^{-\mathbf{1}} \mathbf{U}^{\mathbf{T}}
\end{gathered}
$$

where $\mathbf{Z}_{\mathbf{1}} \in \mathbb{R}^{p \times p}, \mathbf{Z}_{\mathbf{2}} \in \mathbb{R}^{(n-p) \times(n-p)}$ and the singular value decomposition of the matrix $\mathbf{M}$ is represented as $\mathbf{M}=$ $\mathbf{U}\left[\begin{array}{ll}\hat{\mathbf{M}} & \mathbf{0}\end{array}\right] \mathbf{V}^{\mathrm{T}}$ with the unitary matrices $\mathbf{U} \in \mathbb{R}^{p \times p}$, $\mathbf{V} \in \mathbb{R}^{n \times n}$ and a diagonal matrix $\hat{\mathbf{M}} \in \mathbb{R}^{p \times p}$ with positive diagonal elements in decreasing order.

\section{A. $H_{\infty}$ performance}

Under zero initial conditions, the $H_{\infty}$ performance measure can be written as:

$$
\begin{aligned}
J_{\infty} & \leq \int_{0}^{\infty}\left[\mathbf{z}^{\mathrm{T}} \mathbf{z}-\gamma^{2} \mathbf{w}^{\mathrm{T}} \mathbf{w}\right] d t-\left.\mathbf{V}\right|_{t=0}+\left.\mathbf{V}\right|_{t=\infty} \\
& =\int_{0}^{\infty}\left[\mathbf{z}^{\mathrm{T}} \mathbf{z}-\gamma^{2} \mathbf{w}^{\mathrm{T}} \mathbf{w}+\dot{\mathbf{V}}\right] d t
\end{aligned}
$$

Substituting Eq. 7 into Eq. 27 yields the inequality $J_{\infty} \leq$ $\int_{0}^{\infty} \nu^{T} \Pi_{\infty} \nu d s$ where $\nu=\left[\begin{array}{lll}\mathbf{x} & \eta_{\mathbf{d}} & \mathbf{w}\end{array}\right]^{\mathrm{T}}$ and $\Pi_{\infty}$ is given by:

$$
\begin{gathered}
\boldsymbol{\Pi}_{\infty}:=\left[\begin{array}{cc}
\boldsymbol{\Delta}_{\mathbf{1}} & \mathbf{P}^{\mathrm{T}}\left[\begin{array}{c}
\mathbf{0} \\
\mathbf{B}_{\mathbf{1}}
\end{array}\right]+\left[\begin{array}{c}
\overline{\mathbf{C}}^{\mathrm{T}} \mathbf{D}_{\mathbf{1 2}} \\
\mathbf{0}
\end{array}\right] \\
\star & \mathbf{D}_{\mathbf{1 2}}{ }^{\mathrm{T}} \mathbf{D}_{\mathbf{1 2}}-\gamma^{2} \mathbf{I}
\end{array}\right] \\
\boldsymbol{\Delta}_{\mathbf{1}}=\operatorname{sym}\left(\mathbf{P}^{\mathbf{T}}\left[\begin{array}{cc}
\mathbf{0} & \mathbf{I} \\
\overline{\mathbf{A}} & -\mathbf{I}
\end{array}\right]\right)+\left[\begin{array}{c}
\overline{\mathbf{C}}^{\mathrm{T}} \\
\mathbf{0}
\end{array}\right]\left[\begin{array}{c}
\overline{\mathbf{C}}^{\mathrm{T}} \\
\mathbf{0}
\end{array}\right]^{\mathrm{T}}
\end{gathered}
$$

The operator $\operatorname{sym}$ is defined as $\operatorname{sym}(\mathbf{x})=\mathbf{x}+\mathbf{x}^{\mathrm{T}}$. Then, applying the Schur complement lemma on the matrix $\boldsymbol{\Pi}_{\infty}$ (28) yields:

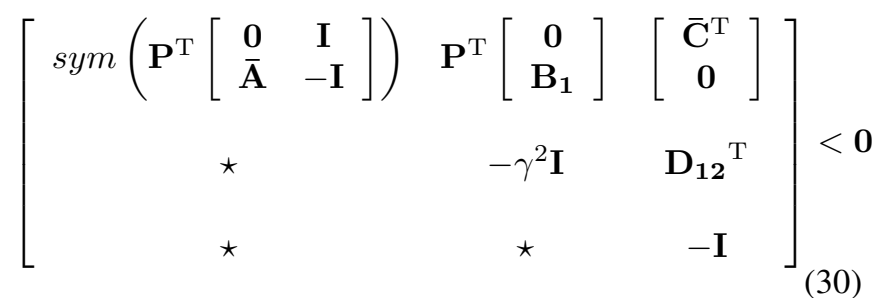

Let $\mathbf{X}=\mathbf{P}^{-1}=\left[\begin{array}{cc}\mathbf{X}_{1} & \mathbf{0} \\ \mathbf{X}_{3} & \mathbf{X}_{\mathbf{2}}\end{array}\right]$ and a congruence transformation $\xi=\operatorname{diag}\left(\mathbf{X}, \mathbf{I}, \mathbf{X}_{\mathbf{1}}\right)$. Pre- and post-multiplying $\xi$ to (30) yields:

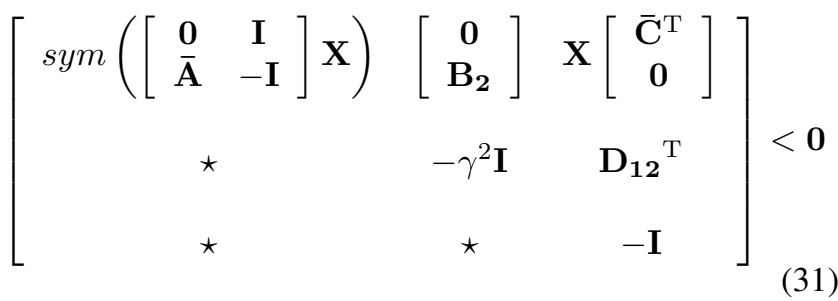


Now consider the equality constraint $\mathbf{C}_{2} \mathbf{X}_{1}=\hat{\mathbf{X}}_{1} \mathbf{C}_{2}$ from Lemma 1 , with $\hat{\mathbf{X}}_{1}$ as a new LMI variable. Let $\tilde{\mathbf{X}}_{\mathbf{1}}=\mathbf{K}_{\mathbf{2} \infty}\left(\mathbf{I}-\mathbf{D}_{\mathbf{2 1}} \mathbf{K}_{\mathbf{2} \infty}\right)^{-1} \hat{\mathbf{X}}_{\mathbf{1}}$. Then, (31) is represented in the following LMI form:

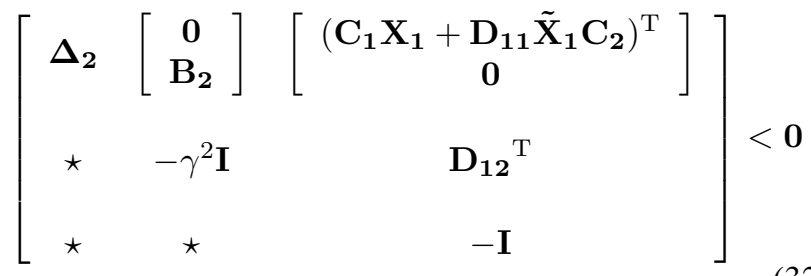

$$
\begin{aligned}
& \boldsymbol{\Delta}_{\mathbf{2}}=\operatorname{sym}\left(\left[\begin{array}{cc}
\mathbf{X}_{\mathbf{3}} & \mathbf{X}_{\mathbf{2}} \\
\mathbf{A} \mathbf{X}_{\mathbf{1}}+\mathbf{B}_{\mathbf{1}} \tilde{\mathbf{X}}_{\mathbf{1}} \mathbf{C}_{\mathbf{2}}-\mathbf{X}_{\mathbf{3}} & -\mathbf{X}_{\mathbf{2}}
\end{array}\right]\right)
\end{aligned}
$$

\section{B. $\mathrm{H}_{2}$ performance}

Recall the Lyapunov function (23). Under zero initial conditions and with $\mathbf{w}=\mathbf{0}$, the $H_{2}$ performance can be written as:

$$
J_{2} \leq \int_{0}^{\infty}\left[\mathbf{x}^{\mathrm{T}} \mathbf{S}_{\mathbf{1}} \mathbf{x}+\mathbf{u}^{\mathrm{T}} \mathbf{S}_{\mathbf{2}} \mathbf{u}+\dot{\mathbf{V}}\right] d t=\int_{0}^{\infty} \nu^{\mathrm{T}} \mathbf{\Pi}_{\mathbf{2}} \nu d t
$$

where the vector $\nu=\left[\begin{array}{ll}\mathbf{x} & \eta_{\mathbf{d}}\end{array}\right]^{\mathrm{T}}$ and the matrix $\Pi_{2}$ is as given in (35).

$$
\begin{gathered}
\boldsymbol{\Pi}_{\mathbf{2}}=\operatorname{sym}\left(\mathbf{P}^{\mathrm{T}}\left[\begin{array}{cc}
\mathbf{0} & \mathbf{I} \\
\overline{\mathbf{A}} & -\mathbf{I}
\end{array}\right]\right)+\boldsymbol{\Delta}_{\mathbf{3}}<\mathbf{0} \\
\boldsymbol{\Delta}_{\mathbf{3}}=\left[\begin{array}{cc}
\mathbf{S}_{\mathbf{1}}+\boldsymbol{\Delta}_{\mathbf{4}}^{\mathrm{T}} \mathbf{S}_{\mathbf{2}} \boldsymbol{\Delta}_{\mathbf{4}} & \mathbf{0} \\
\mathbf{0} & \mathbf{0}
\end{array}\right] \\
\left.\boldsymbol{\Delta}_{\mathbf{4}}=\mathbf{K}_{\mathbf{2} \infty}\left(\mathbf{I}-\mathbf{D}_{\mathbf{2 1}} \mathbf{K}_{\mathbf{2} \infty}\right)^{-1} \mathbf{C}_{\mathbf{2}}\right)
\end{gathered}
$$

Let a congruence transformation $\xi=\operatorname{diag}\{\mathbf{X}, \mathbf{I}\}$. Applying the Schur complement lemma to (35) and then, pre- and post-multiplying $\xi$ to the result yields:

$$
\begin{gathered}
\boldsymbol{\Pi}_{\mathbf{2}}=\left[\begin{array}{cc}
\boldsymbol{\Delta}_{\mathbf{5}} & \mathbf{X}^{\mathrm{T}}\left[\begin{array}{c}
\boldsymbol{\Delta}_{\mathbf{4}}{ }^{\mathrm{T}} \mathbf{S}_{\mathbf{2}} \\
\mathbf{0}
\end{array}\right] \\
\star & -\mathbf{S}_{\mathbf{2}}
\end{array}\right] \\
\boldsymbol{\Delta}_{\mathbf{5}}=\operatorname{sym}\left(\left[\begin{array}{cc}
\mathbf{0} & \mathbf{I} \\
\overline{\mathbf{A}} & -\mathbf{I}
\end{array}\right] \mathbf{X}\right)+\mathbf{X}^{\mathrm{T}}\left[\begin{array}{cc}
\mathbf{S}_{\mathbf{1}} & \mathbf{0} \\
\mathbf{0} & \mathbf{0}
\end{array}\right] \mathbf{X}
\end{gathered}
$$

Let again $\tilde{\mathbf{X}}_{\mathbf{1}}=\mathbf{K}_{\mathbf{2} \infty}\left(\mathbf{I}-\mathbf{D}_{\mathbf{2 1}} \mathbf{K}_{\mathbf{2} \infty}\right)^{-1} \hat{\mathbf{X}}_{\mathbf{1}}$. Then, after substitution of $\mathbf{X}=\left[\begin{array}{cc}\mathbf{X}_{1} & \mathbf{0} \\ \mathbf{X}_{3} & \mathbf{X}_{2}\end{array}\right]$ into (38) and application of the Schur complement to the result, yields the LMI (40).

$$
\left[\begin{array}{ccc}
\boldsymbol{\Delta}_{2} & {\left[\begin{array}{c}
\left(\tilde{\mathbf{X}}_{1} \mathbf{C}_{2}\right)^{\mathrm{T}} \mathbf{S}_{2} \\
\mathbf{0}
\end{array}\right]} & {\left[\begin{array}{c}
\mathbf{X}_{\mathbf{1}}{ }^{\mathrm{T}} \mathbf{S}_{1} \\
\mathbf{0}
\end{array}\right]} \\
\star & -\mathbf{S}_{2} & \mathbf{0} \\
\star & \star & -\mathbf{S}_{1}
\end{array}\right]<\mathbf{0}
$$

The controller gain $\mathbf{K}_{\mathbf{2} \infty}$ can be obtained from the solution of the LMIs (32) and (40) as follows:

$$
\mathbf{K}_{\mathbf{2} \infty}=\left(\mathbf{I}+\tilde{\mathbf{X}}_{\mathbf{1}} \hat{\mathbf{X}}_{\mathbf{1}}^{-1} \mathbf{D}_{\mathbf{2 1}}\right)^{-1} \tilde{\mathbf{X}}_{\mathbf{1}} \hat{\mathbf{X}}_{\mathbf{1}}^{-1}
$$

\section{EXPERIMENTAL RESULTS}

In this section, the mixed $H_{2} / H_{\infty}$ controller is experimentally validated in a Real-Time Hybrid Testing facility at the Smart Structures Technology Laboratory (University of Illinois at Urbana-Champaign, USA). This experimental platform allows for testing large-scale structures through the combination of simulations of numerical models and the experimentation with physical specimens. Usually, the numerical models represent systems whose dynamics are well known such as those of linear systems. On the other hand, the physical specimens are critical components that generally exhibit nonlinear dynamics. Coupling between the physical components and numerical models is done by a software-hardware interface. In this setup, the numerical model corresponds to that of a large-scale three-story building that can be modeled as in (1). The physical specimen is a small-scale magnetorheological (MR) damper.

The experiment consists of exciting the three-story building with seismic motions. The values of the mass, damping and stiffness matrices of this structure are:

$$
\begin{gathered}
\mathbf{M}_{\mathbf{s}}=\left[\begin{array}{ccc}
20253 & 0 & 0 \\
0 & 20253 & 0 \\
0 & 0 & 20253
\end{array}\right] \mathrm{kg} \\
\mathbf{C}_{\mathbf{s}}=\left[\begin{array}{ccc}
7243.2 & -2070 & 0 \\
-2070 & 4138.2 & -2070 \\
0 & -2070 & 2070
\end{array}\right] \mathrm{N}-\mathrm{s} / \mathrm{m} \\
\mathbf{K}_{\mathbf{s}}=\left[\begin{array}{ccc}
9932 & -5661 & 0 \\
-5661 & 11338 & -5661 \\
0 & -5661 & 5661
\end{array}\right] \mathrm{N} / \mathrm{m}
\end{gathered}
$$

The MR damper, which is installed in the first floor of the structure, is used to mitigate the vibrations caused by the earthquake. A picture of the MR damper used in the experiment is shown in Fig. 2. The damping force is produced when the current flowing through the internal coils makes the magnetorheological fluid change its rheological properties. Thus, when no current is flowing, the fluid is in a liquid state, providing low damping force. On the other hand, as the current becomes higher, the fluid goes to a semisolid state and hence, the damping force is higher. In this experiment, the current is generated by a voltage-controlled pulse width modulator (PWM).

The small-scale MR damper dynamics are modeled with the Bouc-Wen model [14]:

$$
\begin{aligned}
f_{m r}^{*} & =\left(c_{m r a}+c_{m r b} v_{f}\right) \dot{x}_{p}+\left(k_{m r a}+k_{m r b} v_{f}\right) x_{p} \\
& +\left(\alpha_{m r a}+\alpha_{m r b} v_{f}\right) \varrho \\
& \varrho \\
& \dot{\omega}=-\varphi\left|\dot{x}_{p}\right| \varrho|\varrho|^{n-1}-\beta \dot{x}_{p}|\varrho|^{n}+\kappa \dot{x}_{p}
\end{aligned}
$$

where $f_{m r}^{*}$ is the damper force, $c_{m r}=c_{m r a}+c_{m r b} v_{f}$ is the voltage-dependent device damping coefficient, $k_{m r}=k_{m r a}+k_{m r b} v_{f}$ is the voltage-dependent device stiffness, $x_{p}$ is the piston displacement, $\varrho$ is an evolutionary 


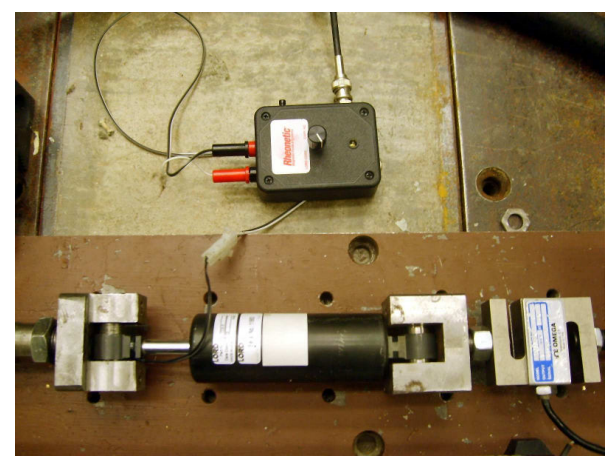

Fig. 2. MR damper and PWM system.

variable that describes the hysteretic behavior of the damper and $v_{f}$ is the voltage output of the first order filter $\dot{v}_{f}=-\eta\left(v_{f}-v\right)$ introduced to represent the transient behavior of the MR fluid in reaching the rheological equilibrium. The parameters of the MR damper specimen are: $\alpha_{m r a}=33.27 \mathrm{~N} / \mathrm{m}, \alpha_{m r b}=182.65$ $\mathrm{N} / \mathrm{m}-\mathrm{V}, c_{m r a}=754.41 \mathrm{~N}-\mathrm{s} / \mathrm{m}, c_{m r b}=712.73 \mathrm{~N}-\mathrm{s} / \mathrm{m}-\mathrm{V}$, $k_{m r a}=1137.57 \mathrm{~N} / \mathrm{m}, k_{m r b}=1443.50 \mathrm{~N} / \mathrm{m}-\mathrm{V}, x_{0}=0$ $\mathrm{m}, \varphi=4209.8 \mathrm{~m}^{-2}, \beta=4205.2 \mathrm{~m}^{-2}, \kappa=10246$, $n=2, \eta=57 \mathrm{~s}^{-1}$. The following scaling factors are used to integrate the physical small-scale MR damper into the numerical large-scale three-story building: the first floor relative displacement is reduced by a factor $S_{L}=7.25$ to obtain the damper piston displacement (that is, $x_{p}=x_{1 r} / S_{L}$ ) and the MR damper force is increased by a factor $S_{F}=60$ to obtain the input force to the structure (that is, $f_{m r}$ in (1) is given by $f_{m r}=S_{F} f_{m r}^{*}$ ).

The details of this system setup and the hardware and software necessary for the interaction between the simulations and the experiment can be found in [13].

The output feedback controller $\mathbf{u}=\mathbf{K}_{\mathbf{2}} \infty \mathbf{y}$ yields a force signal that cannot be commanded to the MR damper. Instead a voltage signal must be generated to drive the damper. In order to implement the controller in this experiment setup, an algorithm was develop to estimate the voltage signal. Let $F_{2 \infty}$ be the control force estimated by the mixed $H_{2} / H_{\infty}$ controller gain. Then, from (45) and (46), the voltage signal to the MR damper is estimated with the following equation:

$$
v=\frac{F_{2 \infty} / S_{F}-\left(c_{m r a} \dot{x_{p}}+k_{m r a} x_{p}+\alpha_{m r a} \varrho\right)}{c_{m r b} \dot{x_{p}}+k_{m r b} x_{p}+\alpha_{m r b} \varrho}
$$

provided that $v \neq 0$, otherwise, $v=0$. The vector of controlled signals is composed of the absolute accelerations and relative velocities of each floor. These signals are weighted by some weighting factors as shown in (11) and (12). Thus, the vector of controlled outputs was is $\mathbf{z}=$

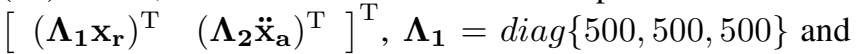
$\Lambda_{\mathbf{2}}=\operatorname{diag}\{500,150,1000\}$. The available measurements are the absolute accelerations of each floor. Finally, the matrices $\mathbf{S}_{\mathbf{1}}$ and $\mathbf{S}_{\mathbf{2}}$ were chosen as $\mathbf{S}_{\mathbf{1}}=\mathbf{I}$ and $\mathbf{S}_{\mathbf{2}}=\mathbf{I}$.
A solution of the set of LMIs (32) and (40) was found with $\gamma=131$. The resulting control gain is:

$$
\mathbf{K}_{\mathbf{2} \infty}=\left[\begin{array}{lll}
493.90 & -4.20 & -95.95
\end{array}\right]
$$

The model of the three-story building and the controller are implemented in Matlab/Simulink. The ordinary differential equation solver used is the $4^{\text {th }}$ order RungeKutta method with a time step $T_{s}=5 \times 10^{-4}$ seconds. The structure is subject to the El Centro, Loma Prieta and Northridge seismic motion records; the scale amplitude used is 0.4 .

The $H_{\infty}$ performance, measured as $\sqrt{\|\mathbf{z}\|} / \sqrt{\|\mathbf{w}\|}$ is shown in Table I for the different seismic motions. The performance bound $(\gamma=131)$ is satisfied the controller under the three seismic motions. In comparison, in the case of no MR damper actuating, the $H_{\infty}$ performance is higher than $\gamma$. Furthermore, the $H_{2}$ performance was measured in all cases. For initial conditions (measured in $\mathrm{cm}$ and $\mathrm{cm} / \mathrm{s}$ )

$\mathbf{x}(\mathbf{0})=\left[\begin{array}{llllll}0.0127 & 0.2032 & 0.0254 & 1.27 & 2.032 & 2.54\end{array}\right]^{\mathrm{T}}$

the bound $J_{0}=\mathbf{x}(\mathbf{0})^{\mathrm{T}} \mathbf{X}_{\mathbf{1}}^{-\mathbf{1}} \mathbf{x}(\mathbf{0})=1.02 \times 10^{9}$ is greater than the $J_{2}$ value achieved by the controllers in all cases, as can be seen in Table II.

\begin{tabular}{ccc}
\hline \hline & Uncontrolled & Mixed $\mathrm{H}_{2} / \mathrm{H}_{\infty}$ control \\
\hline El Centro & 207.15 & 120.14 \\
Loma Prieta & 188.11 & 103.88 \\
Northridge & 182.42 & 113.08 \\
\hline
\end{tabular}

TABLE I

$H_{\infty}$ PERFormance indices Under El Centro, Loma Prieta AND NORTHRIDGE EARTHQUAKES.

\begin{tabular}{cc}
\hline \hline & Mixed $\mathrm{H}_{2} / \mathrm{H}_{\infty}$ control \\
\hline El Centro & $7.29 \times 10^{8}$ \\
Loma Prieta & $4.24 \times 10^{8}$ \\
Northridge & $5.43 \times 10^{8}$ \\
\hline
\end{tabular}

TABLE II

$H_{2}$ Performance indices under El Centro, Loma Prieta AND NORTHRIDGE EARTHQUAKES.

From Fig. 3 it can be seen that the controller is able to reduce the displacement and acceleration responses of the structure. Fig. 4 shows the MR damper response to the input voltage and piston displacement. The resulting damper force is within its reasonable range $( \pm 3000 \mathrm{~N})$. Fig. 5 shows that the controller is also able to reduce the power spectral density of the displacement response of the structure.

\section{CONCLusions}

In this paper, an output feedback mixed $H_{2} / H_{\infty}$ controller was formulated to reduce the vibrations of a 


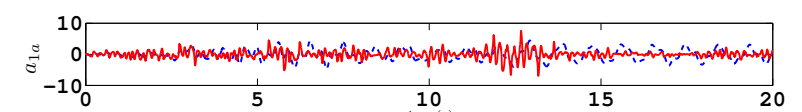

S10

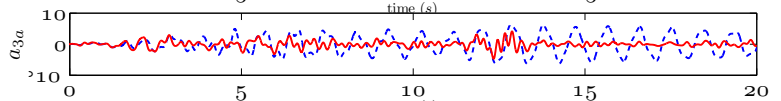

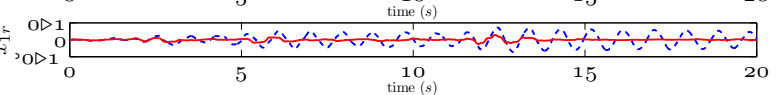

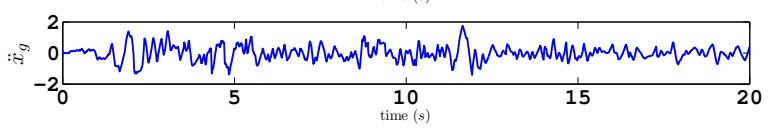

Fig. 3. Acceleration $\left(\mathrm{m} / \mathrm{s}^{2}\right)$ and displacement $(\mathrm{cm})$ response under El Centro earthquake. Dash: uncontrolled; solid: controlled
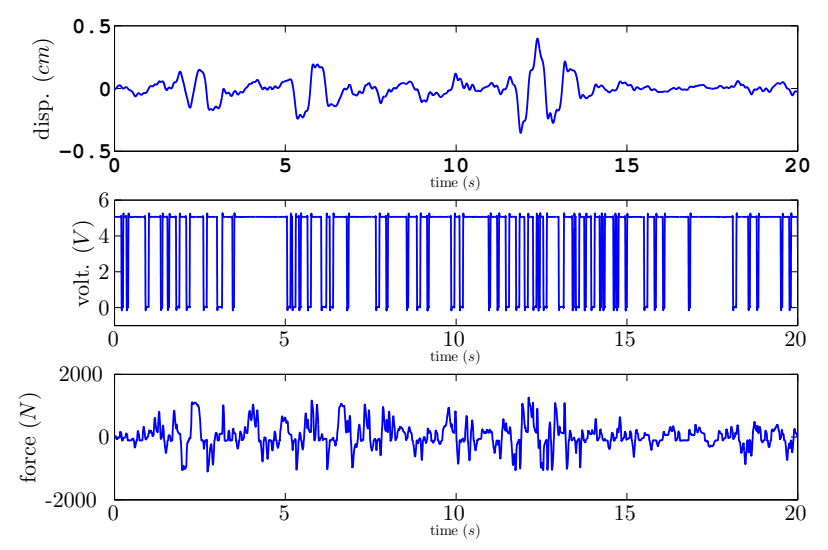

Fig. 4. MR damper response under El Centro earthquake.
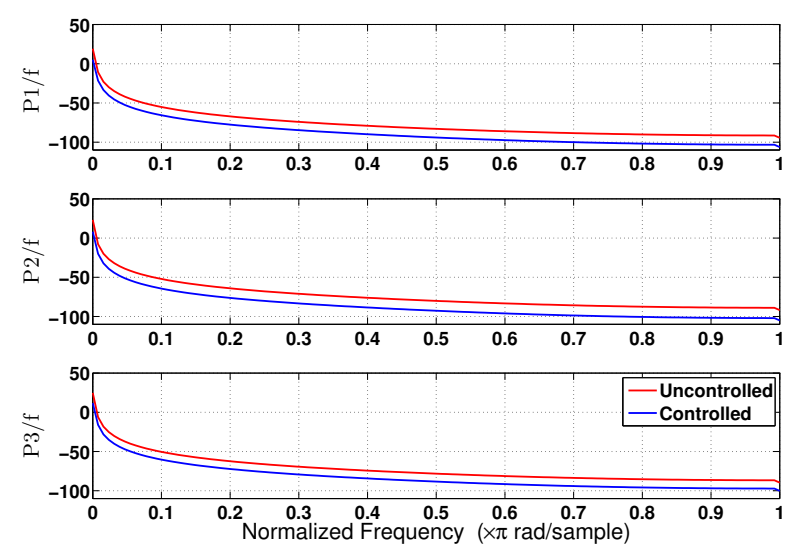

Fig. 5. Displacement power spectral density under El Centro earthquake. three-story building with MR damper when subject to seismic motions. The controller was designed following an LMI approach so that both $H_{2}$ and $H_{\infty}$ performances could be achieved simultaneously. To generate the control signal, an algorithm based on the Bouc-Wen model of the damper was proposed. As a result, the experiments show a reduction in both acceleration and displacement responses. Furthermore, the power spectral density of the displacement of each floor decreased with the action of the controller.

\section{ACKNOWLEDGMENTS}

This work has been partially funded by the European Union (European Regional Development Fund) and the Ministry of Science and Innovation of Spain through the coordinated research projects DPI-2008-06699-C02-01 and by the government of Catalonia through SGR00296. M. Zapateiro acknowledges the FI and BE Grants of the Department for Innovation, University and Enterprise of the Government of Catalonia (Spain). B.M. Phillips is supported under a National Science Foundation Graduate Research Fellowship.

\section{REFERENCES}

[1] B.F. Spencer, Jr., M. Sain, "Controlling buildings: a new frontier in feedback", IEEE Control Systems Magazine, 17, 19-35, 1997.

[2] S.J. Dyke, Y. Wang, E. Taylor, "Seismic response modification using innovative damping devices", Third Colombian Conference on Earthquake Engineering., Cali, Colombia, 2005.

[3] M. Zapateiro, H.R. Karimi, N. Luo, B.F. Spencer, Jr., "Real-time hybrid testing of semiactive control strategies for vibration reduction in a structure with MR damper", Structural Control and Health Monitoring, Available online (DOI: 10.1002/stc.321), 2009.

[4] X. Wang and F. Gordaninejad, "Lyapunov-based control of a bridge using magneto-rheological fluid dampers", Journal of Intelligent Material Systems and Structures,13, 415-419, 2002.

[5] N.H. McClamroch and H.P. Gavin, "Closed loop structural control using electrorheological dampers", American Control Conference, Seattle, Washington, U.S.A., June 21-23, 1995.

[6] S.J. Moon and L.A. Bergman and P.G. Voulgaris, "Sliding mode control of cable-stayed bridge subjected to seismic excitation", Journal of Engineering Mechanics, 129, 71-78, 2003.

[7] F. Pozo and F. Ikhouane and G. Pujol and J. Rodellar, "Adaptive backstepping control of hysteretic base-isolated structures", Journal of Vibration and Control, 12, 373-394, 2006.

[8] S. Narasimhan and S. Nagarajaiah, "Smart base isolated buildings with variable friction systems: $H_{\infty}$ controller and SAIVF device", Earthquake Engineering and Structural Dynamics, 35, 921-942, 2006.

[9] D.J.N. Limebeer, B.D:O. Anderser, B. Hendel, "A Nash game approach to mixed $\mathrm{H}_{2} / \mathrm{H}_{\infty}$ control", IEEE Transactions on Automatic Control, 39, 69-82, 1994.

[10] W. Lin, "Mixed $H_{2} / H_{\infty}$ control of nonlinear systems", $34^{\text {th }}$ Conference on Decision and Control, New Orleans, LA, 1995.

[11] B.S. Chen, W. Zhang, "Stochastic $H_{2} / H_{\infty}$ control with statedependent noise", IEEE Transactions on Automatic Control, 40, 628660, 2001.

[12] D.W.C. Ho, G. Lu, "Robust stabilization for a class of discretetime nonlinear via output feedback: the unified LMI approach", International Journal of Control, 76(2), 105-115, 2003.

[13] J. Carrion, B.F. Spencer, Jr., "Model-based strategies for real-time hybrid testing", Technical Report NSEL-006, University of Illinois at Urbana-Champaign, 2007.

[14] B.F. Spencer, Jr., S.J. Dyke, S. Sain, J. Carlson, "Phenomenological model of a magnetorheological damper", ASCE Journal of Engineering Mechanics, 123, 230-238, 1997. 